\title{
Properties of torsion rod in "Sulzer" projectiles loom
}

\begin{abstract}
The torsion rod characteristics of the Sulzer loom can be adjusted in the zero position by means of the adjusting lever and the adjusting actuator of the torsion rod of loom. In the "Sulzer" projectile loom, the movement of the intermediate member results in the axial movement of the adjusting lever to change the position of the zero according the material and characteristics of rod. The intermediate member transmits a force exerted by the adjusting lever to a fixed sliding surface. However, the described device in some countries has a serious drawback, because the tuning drive acts on the torsion rod adjusting lever so that when the projectile is fired into the "Sulzer" loom, the picking mechanism reacts through the sudden, harmful pressure of the tuning drive. A solid wedge-shaped idler that can be moved by the adjusting motor is placed between the sliding surface of the adjusting lever and a fixed sliding surface. In the "Sulzer" projectile loom, known torsion rod control enables projectile loom operation with improved energy consumption and reduced wear.
\end{abstract}

Volume 8 Issue I - 2022

\author{
ElSayed A EINashar,' Dmitry Pirogov² \\ IProfessor of Textiles \& Apparel, Kaferelsheikh University, Egypt \\ ${ }^{2}$ Ivanovo State Polytechnic University, Ivanovo, Russia
}

Correspondence: ElSayed A EINashar, Professor of Textiles \& Apparel, Kaferelsheikh University, Egypt, Tel +201069288940, EmailSmartex@kfs.edu.eg

Received: February 092022 | Published: February 15, 2022

Keywords: characteristics, torsion rod, projectile, loom, Sulzer

\section{Introduction}

The present properties of torsion rod in projectile loom elates, to a torsion rod assembly for a "Sulzer" projectile of textiles Suspension System, and more particularly to modifying the effective length of the torsion rod in projectile loom to provide variable effective Spring rates. Sulzer are commonly equipped with independent pension Systems which can include a vibration or shock absorbing device for absorbing vibrations shock and other vibrations while providing a smooth and comfortable for the "Sulzer" while working..$^{1-3}$ One type of Shock absorbing System includes a torsion rod. ${ }^{4}$ In Suspension Systems of this type, the torsion rod extends longitudinally along the length of the Sulzer projectile of dynamics picking mechanism of Sulzer projectile loom during its discharge. ${ }^{5}$ The law of motion lever of picking mechanism during its discharge. ${ }^{6,7}$

\section{Analyses of mechanism in torsion rod in "Sulzer"}

Let us consider the technological process of weft insertion and the construction of the fighting mechanism of a STB type loom, which does not differ from the construction of the fighting mechanism of a Sulzer weaving machine. ${ }^{1}$

On STB machines, weft insertion is carried out by small-sized spacers from stationary cross-winding bobbins located on the left side of the machine. Weft spacers are a steel plate with a catch for weft thread. The body of the weft spacer is hollow and has an irregular octagonal prism shape in section. The cone-shaped nose of the pad improves the conditions of the pad flight over the guide comb. Inside the housing, a steel spring is riveted to the rear end with jaws for gripping and holding the filling thread. To open the spring jaws, the weft return spring and the spring expander tooth to transfer the thread to the spacer are included in the opening of the spacer when it is in the take-up box. In the receiver box, the tooth opens the spring to release the thread and transfer it to the yarn collector ${ }^{1}$ Construction of dynamic model the picking mechanism of Sulzer projectile loom. ${ }^{8,9}$

Weft insertion on the machines with small spacers is carried out as follows (Figure 1). Bobbin thread from the bobbin 1 passes through the eye 2 , the yarn collector 3 , peephole 4 , the filling brake 5 , guide eye 6 , the compensator eye 7 , guide eye 8 and is held by the jaws of the filling returner 9. After the transfer of the filling thread from the weft retriever to the weft former 10, it is laid in the shed. The centering device 11 will centre the filling thread in relation to the weft returner. Catchers (clips) 12 approach the edges of each web and clamp the ends of the weft coming out of the edges. Scissors 13 are used to cut the thread. Sometimes an additional tensioning device is installed between the bobbin and the yarn collector. ${ }^{1,10}$

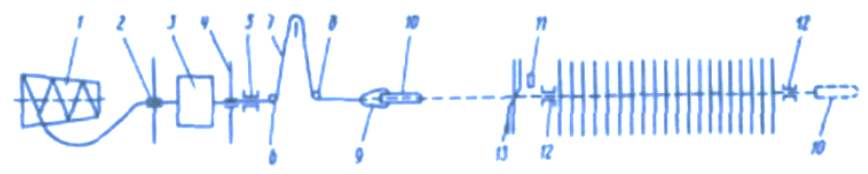

Figure I Weft insertion on the machines with small spacers is carried out.

Laying conditions. The fighting mechanism of weaving looms of STB type differs significantly from the fighting mechanisms of shuttle looms by design and the material from which it is made. ${ }^{2,7,8}$ Acceleration of the shuttle - weft inserter is carried out by the elastic moment of the twisted torsion shaft. The value of the twist angle of this shaft determines the fighting force. Parts of the fighting mechanism are made of alloyed steel, which ensures its reliable and accurate operation. The fighting force of the STB warhead depends on the potential energy of the torsion shaft and does not depend on the rotation speed of the machine's main shaft. The elastic torsion shaft is the main part of the mechanism and has its own characteristics of material, construction, operating parameters and design, ${ }^{3}$ picking mechanism and enters during braking into the cylinder. period of work of picking mechanism consists of four separate of processes that are charging the torsion rod and outputting of mechanism from a dead Charging the torsion rod so its discharge of the torsion rod and acceleration of projectile throw braking of mechanism. ${ }^{6,8}$ The design of the fighting mechanism is shown in Figure 2.

The torsion shaft 1 is clamped at one end in the sleeve 2, attached to the casing 3, which is fixed to the wefted combat box with three screws. Another housing the shaft is placed inside the tube 5.On the end of the tube, opposite the sleeve, mounted combat lever-carrier 19 , at the upper end of it is mounted striker 20 with the projection 21 , which acts on the weft spacer 22 . The torsion shaft is charged with a 
cam 7 , mounted on the drive shaft 17 , which receives the motion from the roller 16. The cam acts on the roller 8 of the three-arm lever10 loosely planted on the axle 14 . The upper arm of the three-arm lever 10 is hinged through the rod 6 to the springs of combat tube 5 . When deflecting the lever 10 tube 5 rotates counterclockwise, twisting the shaft 1 . Swivel lever 10 and twisting the shaft 1 continues until the axis of rotation of the lever 10, rod 6 and lugs combat tube will not be on one straight line (will take the dead position) and the axis of the roller 8 will not depart from the dead position at $0,1-0,2 \mathrm{~mm}$.

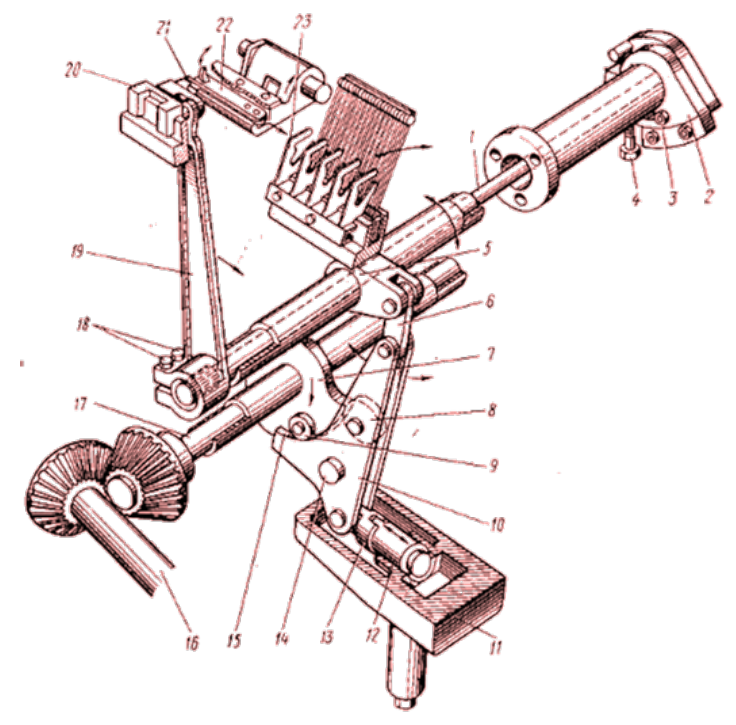

Figure 2 The design of the fighting mechanism.

The breaking force depends on the twist angle of the torsion shaft 1. The twist angle is influenced by the fabric filling width, machine speed, linear density and the type of raw material to be processed. The steepening angle can vary between 26 and $32^{\circ}$. The angle is set with lever bolt 4 . Torsion shaft twist on narrow machines is from 161 to $71^{\circ}$, on wide machines - from 126 to $36^{\circ}$ of rotation of the main shaft. Correspondingly, the moment of engagement should occur at $140 \pm 3$ or $105 \pm 3^{\circ}$ of the main shaft rotation. Start of chattering depends on setting of cam 7 on the shaft 17 . To set the moment of the start of chatter, remove the weft stopper, set the main shaft to the position corresponding to $250^{\circ}$ (for wide machines $220^{\circ}$ ) of the main shaft rotation and unload the torsion shaft. Through the left open window in the lower compartment of the fighting box loosen the bolts, by which the cam 7 is attached to the three-stepped cam, and turn the cam 7 clockwise, when you want to set the beginning of the fight earlier, and vice versa. After that, fasten the cam, wind the torsion shaft and carry out the test run.

The initial velocity of the probe through the shed when the torsion shaft is twisted by $27^{\circ}$ is $19.3 \mathrm{~m} / \mathrm{s}$, and at $32^{\circ}-23.1 \mathrm{~m} / \mathrm{s}$. The flight time of the spacer is equal to 155 or $195^{\circ}$ of rotation of the main shaft, respectively. The speed of movement of the weft insertion hook on narrow and wide machines is approximately the same. The speed required for the hook to pass through the shed is communicated by the fighting mechanism, the operation of which is based on the use of potential energy of the twisted roll $^{4}$ The necessary results can be obtained in a graphical form and carry out automatically analyze the natural frequencies and mode shapes., ${ }^{9,10}$

The torsion roller, depending on the twist angle and its elastic properties, transmits motion through the appropriate levers to the weft inserter, whose equation of motion with a sufficient degree of approximation can be represented in the form. ${ }^{1}$

$$
v_{2}-v_{1}=\frac{1}{m} \int_{0}^{t_{2}}\left(F_{y}+T+Q\right) d t
$$

Where $v_{1}$ - initial velocity of weft layer in free flight, $\mathrm{m} / \mathrm{s} ; v_{2}$ - final velocity of weft layer in free flight, $\mathrm{m} / \mathrm{s} ; F_{y}$ - tension of laid filling thread, $\mathrm{N} ; T$ - reduced friction force of weft layer against channel guide teeth, $\mathrm{N} ; Q$ - force of frontal air resistance at weft layer movement in shed, N.

Assuming that the motion of the laying machine is uniformly slow and the forces acting during laying are constant, we obtain:

$$
v_{2}-v_{1}=\frac{1}{m}\left(F_{y}+T+Q\right) t_{2}
$$

Where $t_{2}$ is free flight time of weft insertion, $\mathrm{s}$.

The fighting mechanism gives the weft pusher a maximum speed when flying in the shed. This velocity during laying is reduced by the forces listed above. The air drag force can be determined by the formula.

$$
Q=W \cdot S \cdot v_{\tilde{n} \delta}^{2} \cdot p,
$$

Where $W$ - drag coefficient; $S$ - frontal area of weft laying, $\mathrm{m}^{2} ; v_{c p}$ average speed of weft laying in free flight, $\mathrm{m} / \mathrm{s} ; p$ - air density, $\mathrm{kg} / \mathrm{m}^{3}$.

Calculations show that the air resistance force is a few centinewtons. The weft pacer experiences friction against the guide teeth during the period of movement through the shed. The reduced friction force of the weft insert can be determined by formula 4

$$
T=f_{i \delta} \cdot\left(G+P_{i}\right)
$$

Where $f_{n p}$ - reduced coefficient of friction of weft laying on channel guide teeth; $G$ - mass of weft laying, $\mathrm{kg} ; P_{i}$ - normal component of weft laying inertia force, N. Calculations show that this force is small, so the weft stretching influences on weft laying deceleration in the shed to the greatest extent.

In general terms, the law of motion of the fighting mechanism, not taking into account the elastic deformations of all parts of the fighting mechanism except for the torsion shaft, since their deformations during acceleration and deceleration are small, can be described during operation using the Lagrangian equation of the second kind ${ }^{2}$ as follows.

$$
J_{r e d}(\varphi) \cdot \varepsilon+\left(\frac{\omega^{2}}{2}\right)\left[\frac{d J_{r e d}(\varphi)}{d \varphi}\right]=M_{m o v}(\varphi)-M_{r e s}(\varphi),
$$

Where $J_{\text {red }}(\varphi)$ - mass moment of inertia of the moving parts of the mechanism reduced to the axis of the torsion shaft; $\varphi$ - is the generalized coordinate, in this case the angle of rotation of the torsion shaft from the zero position (twist angle); $\varepsilon u \omega$ - is the angular acceleration and angular velocity of the drive element, i.e. the torsion shaft $M_{m o v}$ - is the driving torque of external forces applied to the axis of the torsion shaft $M_{\text {res }}$ - Resistance torque reduced to the torsion shaft axis, i.e. the torque from friction forces in the supports and guide links, from elastic forces of the twisted shaft, from hydraulic resistance forces of the oil brake.

The acceleration period of the paver is the most important period for this process. During this period the driving moment of external forces $M_{m o v}$ is zero and the drag forces are insignificant. At this 
point in time, the mechanism moves under the action of the elastic momentum of the torsion shaft. During the acceleration period, the combat mechanism moves in accordance with the equation

$$
J_{r e d}(\varphi) \cdot \varepsilon+\left(\frac{\omega^{2}}{2}\right)\left[\frac{d J_{r e d}(\varphi)}{d \varphi}\right]=M_{e l}(\varphi),
$$

ãäå $M_{e l}(\varphi)=c_{t} \cdot \varphi$, Elastic torque of the torsion shaft, $c_{t}-$ is the torsional stiffness coefficient of the torsion shaft.

Since the elastic momentum is a function of the generalized coordinate $\varphi$, solving (6) with $\omega$, we obtain

$$
\omega=\sqrt{G \frac{\left(\varphi_{0}{ }^{2}-\varphi^{2}\right)}{J_{\text {red }}(\varphi)}+\frac{\omega_{0}{ }^{2} \cdot J_{\text {red }}\left(\varphi_{0}\right)}{J_{\text {red }}(\varphi)}}
$$

Where $\varphi_{0}, \varphi$ - are the initial and current angles of twist of the torsion shaft during its acceleration (counting $\varphi_{0}, \varphi$ is taken from the zero position) $J_{\text {red }}\left(\varphi_{0}\right), J_{\text {red }}(\varphi)$ - the moment of inertia of masses of moving parts reduced to the axis of the torsion shaft, respectively for the initial and current positions of the mechanism at its acceleration $\omega_{0}$ - is the speed of the torsion shaft at the beginning of its acceleration, obtained when the mechanism is removed from its rest position.

The masses and moments of inertia of the moving parts are reduced to the axis of the torsion shaft based on the equivalence of the kinetic energy of the mechanism and the reduced system.

Torsional shaft elastic moment

$$
M_{e l}(\varphi)=c_{t} \cdot \varphi=\frac{G J_{p}}{L} \cdot(\varphi)
$$

Where $G$ - is the second kind modulus of elasticity of the torsion shaft material; $J_{p}$ - is the polar moment of inertia of the torsion shaft cross section, $L$ is the length of the torsion shaft twisted section.

Knowing $\omega$, it is possible to determine the acceleration speed of the paver

$$
V_{i}=\omega \cdot y(\varphi)
$$

Where $y(\varphi)$ - is the transfer function from the torsion shaft to the spacer.

\section{Results and discussions}

The modern techniques of weft insertion in projectile weaving machine, Due to this the weft is inserted at substantially higher speed. The projectile has clamps at one end which will hold the weft yarn. The theoretical speed of the spacer increases in time as the torsion shaft unwinds and reaches its maximum value by the end of acceleration. ${ }^{2}$ Engineers in industry and education schools use the tool for the design, analysis and virtual testing of complex mechatronics systems, ${ }^{8,9}$ the runner to be accelerated is detached from the race at the moment when the oil brake begins to act when the torsion shaft unwinds by about $14^{\circ}$, reaching a speed of $22-24 \mathrm{~m} / \mathrm{s}$. The theoretical variations of velocity $V_{u}$ of the spacer and the chase $V_{n}$ on STB type machines are shown in Figure 3.

To ensure reliable operation of the combat mechanism of STB type machines it is necessary that the oil brake fully absorbs all kinetic energy of the combat mechanism after acceleration of the weft laying head. Changing the operation of the oil brake leads to corresponding changes in the braking force, which can cause dynamic shocks in the joints and joints of the mechanism and, on the other hand - with too sharp braking - the emergence of large drag forces, and as a consequence, large stresses in the parts of the mechanism. Theoretical studies have shown that the oil brake of STB type machines allows carrying out braking within 3-5 ms, but this value depends significantly on the oil viscosity, which in turn depends on the temperature and operating time.

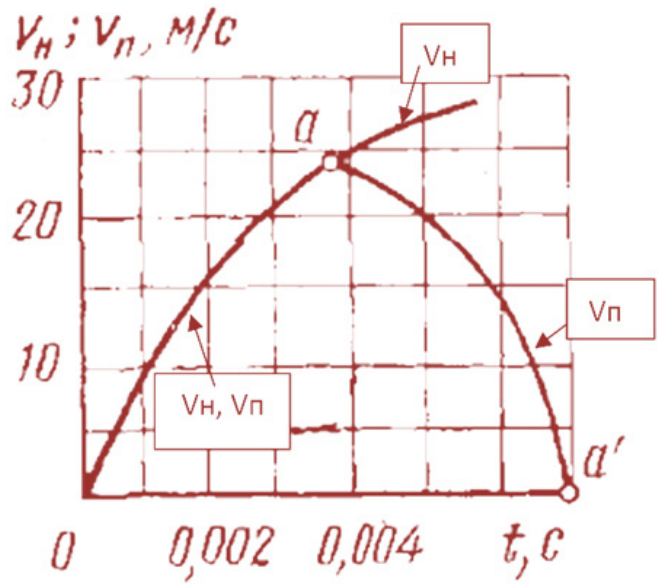

Figure 3 The theoretical variations of velocity $V_{H}$ of the spacer and the chase $V_{n}$ on STB type machines.

This projectile gets the motion due to the torsion rod picking mechanism. In this mechanism the picking cam attached to the picking shaft which is getting the drive from the main shaft through bevel gear. Therefore once the roller attached to a picking cam is no longer in contact with the toggle plate suddenly the torsion rod gets untwisted with a lot of energy. This generated energy is then transferred to the projectile through a picking arm and picking shoe.

As the analysis of the results of theoretical and experimental studies shows, the efficiency of the combat mechanism of STB type machines is about $25 \%$, which means that as a result only a small part of its energy is used for acceleration of the paver. Therefore, this mechanism needs improvement and modernization in order to increase its efficiency.

This picking cam has the roller attached to it. Once the roller is in contact with the toggle plate, it gives the motion to plate. The toggle plate is further connected to the torsion rod with short linkage. The torsion rod is fixed at one end and another end is free to rotate around its axis. Due to the motion given to the toggle plate the torsion rod gets twisted since the opposite end is fixed. This torsion rod has the extraordinary properties to regain its original shape without any permanent deformation.

\section{Conclusion}

Properties of torsion rod in projectiles loom in analysis of this article, the three properties of torsion rod in projectiles loom were subjected to various tests. the properties analysis of previous literature studies investigations and stress calculations were performed, projectile visual inspection and fractography, hardness torsion rod tests, chemical torsion rod analysis, metallographic between torsion rod analysis, and projectiles torsional stress calculation into projectiles loom.

Techniques torsion rod in projectiles loom used in fracture analysis between torsion rod and projectiles the continuing torsion 
rod in projectiles loom of torsion bars may include a design error, an application and a manufacturing. The main disadvantage of projectile weaving technique is that the energy utilization is not efficient than the shuttle loom. About $62 \%$ of the energy in the picking system is absorbed by the oil brakes wasted. This disadvantage is eliminated by the modern techniques of weft insertion in projectile weaving machine, along with some improvements in speed, productivity and flexibility.

Torsion rod and projectiles the design include factors such as improper shoulder geometry torsion rod and projectiles and improper materials selection. Application of torsion rod and projectiles can be including mounting, installation, and maintenance and heat treatment processes.

\section{Acknowledgments}

None.

\section{Funding}

None.

\section{Conflicts of interest}

Authors declare that there is no conflict of interest.

\section{References}

1. Nikolaev SD, Vlasov PV. And other theory of processes, technology and equipment of weaving production. In: Nikolaev CD, Vlasov PV, Sumarukova RI, editors. 2nd edn. M.: Legprombytizdat.1995. $256 \mathrm{p}$ ISBN 5-7088-0410-6.
2. Ditsky AV. Basics of designing weaving machines: Textbook for students. In: Ditsky AV, Malafeev RM, Terentyev VI, editors. Moscow: Mashinostroenie; 1983.320 p.

3. Berdnikov Andrey Yurievich. Development, research and introduction of methods and equipment for technical diagnostics of torsion shafts of weaving looms of STB type: Ph.D thesis. 1984. 168 p.

4. Gordeev NI. To the calculation of the design initial speed of the plower of high-speed weaving machines of the STB type. Textile industry technology. 1989:90-93.

5. Elsayed Elnashar. Design of database for forecasting the specification of woven fabric design for ladies dresses.Ph.D thesis, Cairo, Egypt: University of Helwan; 2000.

6. Elsayed Elnashar. Effect of warp-ends densities distributions on some esthetical and physical properties of multi- layers woven fabric" Msc. Thesis. Faculty of applied arts, Cairo, Egypt: University of Helwan; 1995.

7. Elsayed A, Elnashar. Applications of mechatronics opportunities in textiles. International Robotics \& Automation Journal. 2022.

8. ElSayed A ElNashar. Sustainable textiles industries in brand technology between technologies of brands". 3rd International Webinar Advanced Materials December 13 2021, International Conference on Advanced Materials, Materials Science and Nanomedicine, Digital Copy of the Conference Proceeding. 2021.

9. El Sayed A Elnashar, GV Bashkova. Integration factor construction to improving aesthetic, physical and mechanical properties of double layer woven fabrics by denting and stitches systems. Textile Sci \& Fashion Tech. 2018;1(2).

10. ElSayed A ElNashar. Smart textile circuitry and there application. Current Trends in Fashion Technology \& Textile Engineering. 2018;4(2). 